\title{
A Case Giving Proof of Pathogenesis of Abdominoscrotal Hydrocele: A Case Report
}

\author{
Hee Jo Yang ${ }^{1}$, Myoung Won Son ${ }^{2}$, Doo Sang Kim ${ }^{1}$ \\ Departments of 'Urology and ${ }^{2}$ Surgery, Soonchunhyang University Cheonan Hospital, Soonchunhyang University College of Medicine, Cheonan, Korea
}

\begin{abstract}
An abdominoscrotal hydrocele (ASH) is an uncommon condition characterized by cystic mass that occupies the scrotum and abdomen. The characteristic aspect is the presence of two hydrocele sacs in the abdominal cavity and the scrotum, and compression of one side causes enlargement of the other side, thereby connecting the two sacs. There are several hypotheses regarding the occurrence of ASH. However, the evidence of the hypotheses is lacking. The patient was a 52-year-old male. During observation of the right inguinoscrotal hydrocele, the patient complained of a palpable mass lesion on the right lower quadrant of the abdomen. Physical examination revealed ASH that occurred with the growth of the inguinoscrotal hydrocele. This was further confirmed by sequential imaging test thereby proposing the verification of cephalad extension of hydrocele. The surgical excision was recommended and inguinal exploration was performed under general anesthesia. No evidence of relapse was observed during the 2-year follow-up period after surgery. The present case proves the cephalad extension of hydrocele among other hypotheses on the etiology of ASH.
\end{abstract}

Keywords: Testicular hydrocele; Etiology; Surgical procedure

\section{INTRODUCTION}

An inguinoscrotal hydrocele is a common disease occurring in both children and adults. However, abdominoscrotal hydrocele (ASH) in which the hydrocele is expanded into the abdomen through a deep inguinal ring and forms a dumbbell shape is uncommon. Initially, the number of ASH reports was small because the diagnosis was made only by physical examination or surgery. Currently, numerous cases of ASH have been reported since the spread of ultrasonography $[1,2]$. The exact mechanism underlying ASH is still unclear. However, the most commonly proposed theories are as follows: (1) cephalad extension of a simple hydrocele, (2) high obliteration of processus vaginalis, (3) and processus vaginalis acting as a one-way valve with cephalad extension of hydrocele sac [3]. We report a case of ASH that explains the underlying mechanism through serial computed tomography (CT) imaging.

\section{CASE REPORT}

A 52-year-old man visited urology department with right scrotal swelling for the past 6 months. He was undergoing a regular medical check-up at the department of gastroenterology for liver cirrhosis (LC). The recent increase in mass size was the drawback of manual reduction of palpable inguinoscrotal mass. The incarcerated inguinal hernia was suspected, and an abdomen CT scan was performed (Fig. 1). The patient was diagnosed with an inguinoscrotal hydrocele and was lost to follow-up. After 1 year and 6 months, the patient visited again as the size of the previous mass had increased and another palpable mass appeared in the right lower quadrant of the abdomen.

Physical examination revealed continual non-transilluminant, very large, and tense right scrotal swelling in the lower abdomen. The right testis could not be palpated separately from the swelling. There was a tense, cystic hypogastric lump that had cross fluctua- 

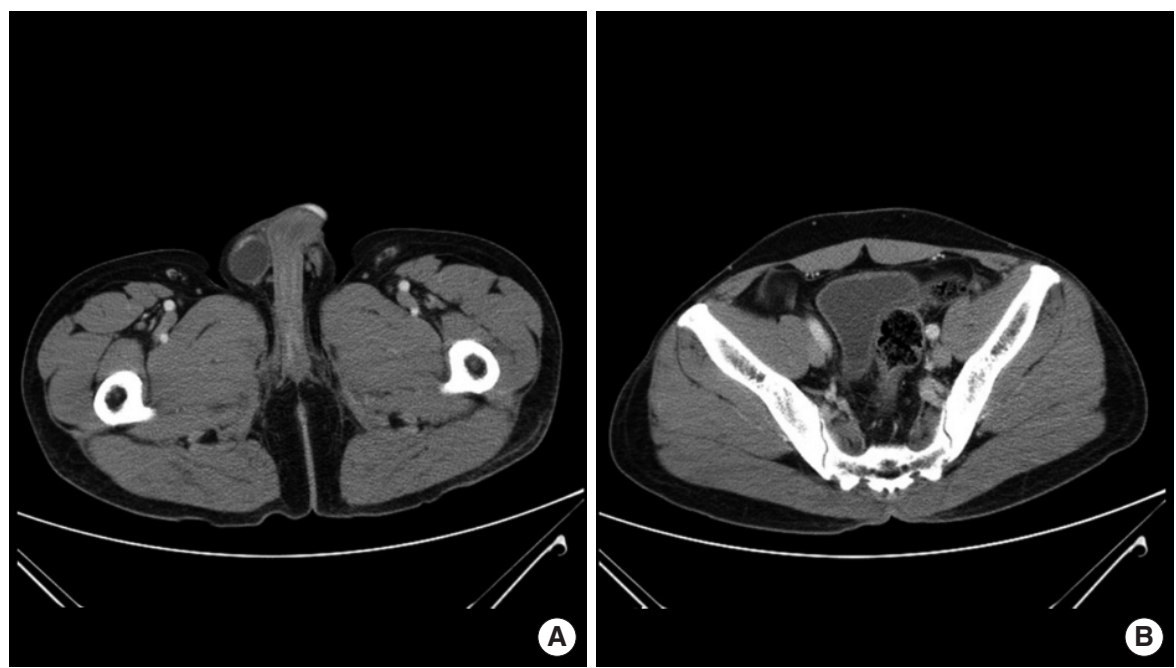

Fig. 1. (A) Computed tomography shows the right inguinoscrotal hydrocele. (B) There was no abdominal component.
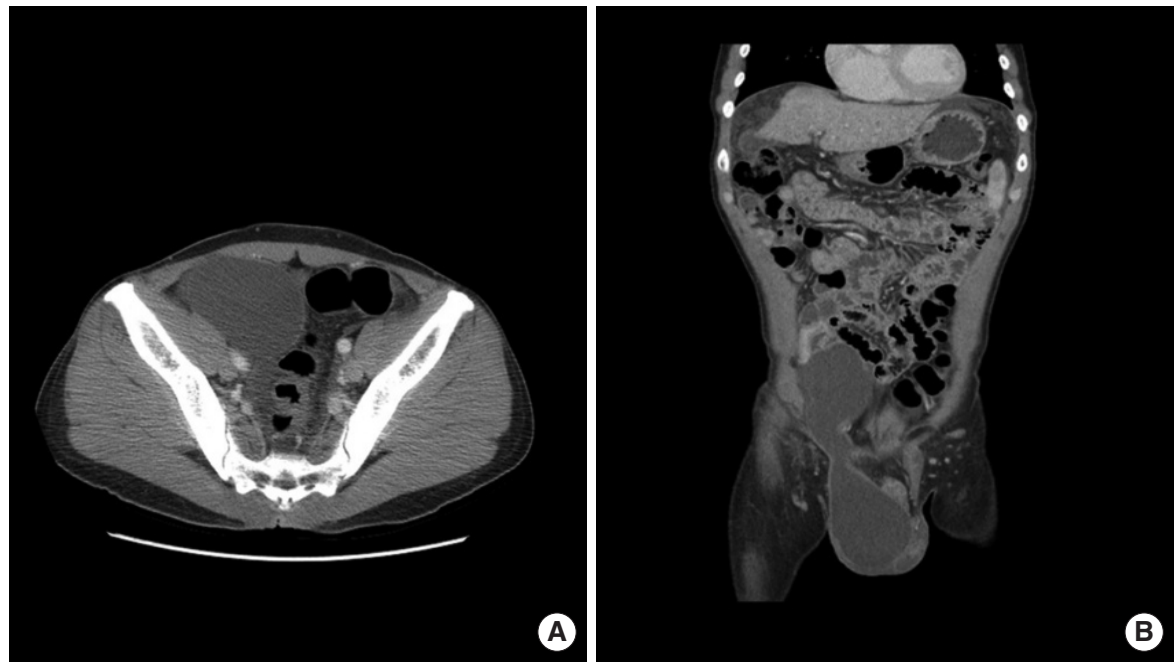

Fig. 2. Computed tomography after 1 year. (A) The scan shows the abdominal component of the abdominoscrotal hydrocele. (B) The coronal image demonstrates an hourglass-shaped abdominoscrotal hydrocele.

tion with the inguinoscrotal hydrocele.

The clinical diagnosis of the ASH was made and confirmed by a CT scan. Contrast-enhanced CT of the abdomen and pelvis showed a well-defined lower abdominal cyst extending into the scrotum through the inguinal canal (Fig. 2).

The surgical excision was recommended and inguinal exploration was performed under general anesthesia. Through an inguinoscrotal incision, the cyst was decompressed, and the abdominal component was dissected free from the retroperitoneum through the widened deep ring. The inguinoscrotal component was completely excised along with cord structures although there was some adhesion to the surrounding tissues. The spermatic cord and testis were normal. Tension-free anterior inguinal repair was performed with a polypropylene mesh, taking into account the slack fascia transversalis and the weakened deep inguinal ring.

Our study was approved by the Soonchunhyang University Hospital Institutional Review Board (IRB approval no., 2020-10014). The patient has provided informed consent for publication of the case.

\section{DISCUSSION}

ASH is a hydrocele that forms two interconnected sacs filled with fluid in the scrotal and abdominal cavity. It often begins with 
an inguinoscrotal hydrocele that extends into the abdomen at birth. It is sometimes found and diagnosed after the patient becomes an adult [2]. Abdominal and scrotal sacs are connected through an inguinal canal resulting in a dumbbell shape. The size of the abdomen and scrotal hydrocele is independently variable. Usually, the abdominal sac is larger than the scrotum and the free cross-movement between the two pockets is characteristics [4]. This is the diagnostic finding for clinical and imaging tests.

Since its introduction, reports of ASH have increased in the literature with an increase in awareness about the disease and widespread application of ultrasonography $[1,2]$. The diagnosis comprises a "spring back ball sign" that compresses the component of the scrotal sac and promotes the expansion of the hydrocele into the abdomen [5]. CT or magnetic resonance imaging may be helpful for distinguishing anatomically complex structure or when the diagnosis is ambiguous in conventional examinations. These tests can be useful for an enhanced description of the overall shape of the ASH, as well as the relationship with or impact on the surrounding structures. In the present case, the patient visited the department with suspicious inguinal hernia, and CT was taken to distinguish strangulated hernia. Afterwards, he was lost to followup and came back after 1 year and 6 months with aggravated symptoms. Compared to the previous physical examination, the size of the inguinal mass had increased and the manual reduction was failure, so CT was again taken to confirm the image with the previous one.

Various theories about the etiology of ASH have been suggested, but are not yet fully understood. The three most commonly proposed theories are as follows: (1) cephalad extension of simple hydrocele by increasing intra-hydrocele pressure, (2) high extinction of patent processus vaginalis (PPV), and (3) PPV acting as a oneway valve with a cephalad extension of the hydrocele [3]. Guillaume Dupuytren, who first reported about ASH, suggested that with an increase in the size of the hydrocele, the pressure in the hydrocele sac increases, and internal pressure progresses through the inguinal canal into the abdomen, thereby causing displacement of the hydrocele $[5,6]$. Some authors have suggested that ASH is caused by the opening of the processus vaginalis, which must act as a valve, and obstruction in the high level, thereby resulting in the pressure in the inguinoscrotal hydrocele against the occlusion point on the internal inguinal ring leading to intra-abdominal expansion [2]. Finally, the discovery of PPV and hydrocele sac in the inguinal canal suggested the possibility that PPV acts as a one-way valve between the hydrocele and the abdominal cavity $[7,8]$. This is based on the fact that processus vaginalis proceeds in parallel with ASH, and ASH rarely resolves spontaneously so that the fluid is replenished into the abdominal cavity. In particular, the authors assumed that inflammation of the tissue surrounding processus vaginalis could trigger a one-way valve mechanism [8]. To support the given theory, the results of their surgery were presented. There exist difficulties in removing the ASH due to adhesion during surgery leading to complications in securing an accurate surgical field; however, the scenario is subjective to the operator's field of view and judgment.

In this case, the progress of ASH was confirmed based on an increase in the frequency of advanced imaging and clinical observation. The ilioinguinal hydrocele was first observed on physical examination and initially, abdominal expansion was not confirmed on CT. The causes of primary hydrocele are lymphatic hypoplasia, lymphatic obstruction (secondary to the presence of inguinal hernia, minor trauma, chronic epididymitis, filaria worms, or a highligature operation for varicocele). In addition, imbalance between the rate of exudation of fluid in the tunica vaginalis and its absorption are the cause of hydrocele [9]. In our patient, LC was well controlled. And even when ASH occurs, there was no deterioration of LC. So, it is unlikely that ASH is caused by LC. After a certain period, the hydrocele had moved from the deep groin ring into the abdomen. It remains unclear why ASH occurs in only a part of the inguinoscrotal hydrocele. Perhaps the second or third hypothesis stated above could be a reason. We propose that frequent physical and imaging tests are needed to clarify this occurrence.

We found that the progress from simple inguinoscrotal hydrocele to ASH with serial CT scans. These observations can be the actual mechanism behind ASH development and support the Dupuytren's theory (cephalad extension of simple hydrocele).

\section{CONFLICT OF INTEREST}

No potential conflict of interest relevant to this article was reported.

\section{ACKNOWLEDGMENTS}

This research was supported by the Soonchunhyang University Research Fund. 
Yang $\mathrm{HJ}$, et al. • A Pathogenesis of ASH, Cephalad Extension of a Simple Hydrocele

\section{REFERENCES}

1. Sasidharan P, Crankson S, Ahmed S. Fetal abdominoscrotal hydrocele. Am J Obstet Gynecol 1991;165(5 Pt 1):1353-5.

2. Cuervo JL, Ibarra H, Molina M. Abdominoscrotal hydrocele: its particular characteristics. J Pediatr Surg 2009;44:1766-70.

3. Doudt AD, Kehoe JE, Ignacio RC, Christman MS. Abdominoscrotal hydrocele: a systematic review. J Pediatr Surg 2016;51:1561-4.

4. Jamal YS, Jamal HS, Al Rahman JS. Abdomino-inguino-scrotal hydrocele (ten-hydroceles with intraabdominal extension). J King Abdulaziz Univ Med Sci 2004;11:49-59.
5. Wlochynski T, Wassermann J, Generowicz Z. Abdominoscrotal hydrocele in childhood. J Pediatr Surg 1993;28:248-50.

6. Bayne A, Paduch D, Skoog SJ. Pressure, fluid and anatomical characteristics of abdominoscrotal hydroceles in infants. J Urol 2008;180(4 Suppl): 1720-3.

7. Khalili M, Gholamzadeh Baeis M, Rouzrokh M. Abdominoscrotal hydrocele: a case report. Urol Case Rep 2020;32:101254.

8. Avolio L, Chiari G, Caputo MA, Bragheri R. Abdominoscrotal hydrocele in childhood: is it really a rare entity? Urology 2000;56:1047-9.

9. Abdel-Rheem MS. Cause of primary vaginal hydrocele and ascites in advanced liver cirrhosis. Am J Surg 1983;146:647-51. 